\title{
Silent hypoxia in COVID-19: pathomechanism and possible management strategy
}

\author{
Ahsab Rahman ${ }^{1} \cdot$ Tahani Tabassum $^{1} \cdot$ Yusha Araf ${ }^{2} \cdot$ Abdullah Al Nahid $^{3} \cdot$ Md. Asad Ullah ${ }^{4} \cdot$ Mohammad Jakir Hosen $^{2}$
}

Received: 9 January 2021 / Accepted: 16 April 2021 / Published online: 23 April 2021

(c) The Author(s), under exclusive licence to Springer Nature B.V. 2021

\begin{abstract}
The novel coronavirus disease 2019 (COVID-19) has become a severe health issue, especially to the patients who develop silent hypoxia condition after SARS-CoV-2 infection. Due to the lack of dyspnoea and extremely low oxygen saturation level, these patients are at exceptionally higher risk. Although the prevalence of silent hypoxia in COVID-19 patients has been evident in several cases, the underlying pathomechanism behind this condition is still unclear. Silent hypoxia in SARSCoV-2 infected patients can be diagnosed with the help of a pulse oximeter, blood gas levels, and a 6-min walking test. While the clinicians and researchers figure out the exact reason for this phenomenon, the patients must be under strict day-to-day monitoring. In this article, we aim to provide comprehensive insights into the underlying symptoms, mechanism, and possible factors behind the occurrence of silent hypoxia among COVID-19 patients.
\end{abstract}

Keywords Silent hypoxia $\cdot$ COVID-19 $\cdot$ Management $\cdot$ Pathomechanism $\cdot$ Dyspnoea

$\begin{array}{ll}\begin{array}{l}\text { Abbreviations } \\ \text { COVID-19 }\end{array} & \begin{array}{l}\text { Coronavirus disease 2019 } \\ \text { SARS-CoV-2 }\end{array} \\ & \begin{array}{l}\text { Severe acute respiratory syndrome } \\ \text { coronavirus-2 }\end{array} \\ \text { 6MWT } & \text { 6-Minute walking test } \\ \text { HIF } & \text { Hypoxia-inducible factor } \\ \text { pRBC } & \text { Packed red blood cell transfusion } \\ \text { ICS } & \text { Inhaled corticosteroids } \\ \text { LDH } & \text { Lactate dehydrogenase } \\ \text { ACE2 } & \text { Angiotensin-converting enzyme 2 }\end{array}$

Ahsab Rahman, Tahani Tabassum, Yusha Araf authors contributed equally to this work

Mohammad Jakir Hosen

jakir-gen@ sust.edu

1 Biotechnology Program, Department of Mathematics and Natural Sciences, School of Data and Sciences, Brac University, Dhaka, Bangladesh

2 Department of Genetic Engineering and Biotechnology, School of Life Sciences, Shahjalal University of Science and Technology, Sylhet, Bangladesh

3 Department of Biochemistry and Molecular Biology, School of Life Sciences, Shahjalal University of Science and Technology, Sylhet, Bangladesh

4 Department of Biotechnology and Genetic Engineering, Faculty of Biological Sciences, Jahangirnagar University, Dhaka, Bangladesh

\section{Introduction}

The "Coronavirus Disease-2019" (COVID-19) outbreak has become an issue of global concern due to its easily transmissible nature [1]. Emerging from Wuhan in December 2019, the disease has so far affected 215 countries around the globe; 133 million people have been infected and 2,892,333 individuals have died [2]. This disease is caused by the severe acute respiratory syndrome coronavirus-2 (SARSCoV-2), which targets the human respiratory system and can induce multiple organ failure. Respiratory damage can give rise to a plethora of health issues in an infected patient, including silent hypoxia [3-7]. Silent hypoxia is defined as a condition where an individual has alarmingly lower oxygen saturation level than anticipated ( 50-80\% saturation, while the anticipated saturation level is $95 \%$ or higher), however, the individual does not experience any breathing difficulty [8]. Normally, an increased respiratory rate (tachypnoea) is seen in normal hypoxic patients, but it is not typically found in patients with silent hypoxia [9-15]. Several reports revealed that the prevalence of silent hypoxia in COVID19 patients ranges from 20 to $40 \%$ [10, 16-18]. In a separate study, a 6-min walking test (6MWT) was applied in a cohort of discharge-ready COVID-19 patients where 50\% of the patients developed exercise-induced hypoxia without dyspnoea [19]. Thus, it is evident that COVID-19 patients can suffer from silent hypoxia which will not induce any 
shortness of breathing initially but might incur rapid damage within the patients, however, the underlying pathomechanism of silent hypoxia occurrence in COVID-19 patients is still not properly understood (Fig. 1).

\section{Underlying factors associated with silent hypoxia in COVID-19 patients}

Existing research findings revealed that the possible reasons for lack of dyspnoea in silent hypoxic COVID-19 patients are- (i) silent hypoxic COVID-19 patients having a slightly increased level of carbon dioxide $(\sim 34-41 \mathrm{~mm} \mathrm{Hg}$, while the normal blood carbon dioxide concentration is $\sim 22-29 \mathrm{~mm}$ $\mathrm{Hg}$ ). The alteration in carbon dioxide level triggers a hypoxia threshold resulting in precipitation of the lung damage indicator of dyspnoea symptoms. Whereas in normal hypoxic conditions, even minute imbalance in the $\mathrm{PaCO}_{2}$ levels rapidly evokes large increases in minute ventilation and brief respiratory alkalosis, which provokes dyspnoea. However, normal hypoxia-induced dyspnoea does not evoke a stronger and excessive volume like hypercapnia does [17]. This might be the reason for COVID-19 patients not showing any sign of distress even during the low oxygen saturation level [10]; (ii) the virus having an impact on the brain and nervous system; altering the mechanisms in our brain responsible for regulating respiration[10]; (iii) the virus having an effect on blood vessels and causing "lack of hypoxic vasoconstriction" [7]; (iv) undergoing hyper-fusion within a lung that has already gone through respiratory damage [11]; (v) fever caused by COVID-19 having a role in silent hypoxia [12], although there is not enough evidence to support this theory (Fig. 2).

\section{Mechanism of silent hypoxia and COVID-19}

An optimum oxygen homeostasis is an essential event for the precise cell system regulation of invertebrates. Any alternation of this oxygen level might be stressful for our bodily functions and can lead to the activation or regression of several homeostasis regulatory genes. Hypoxia can drastically damage several organs and play a crucial role in severe pathological conditions.

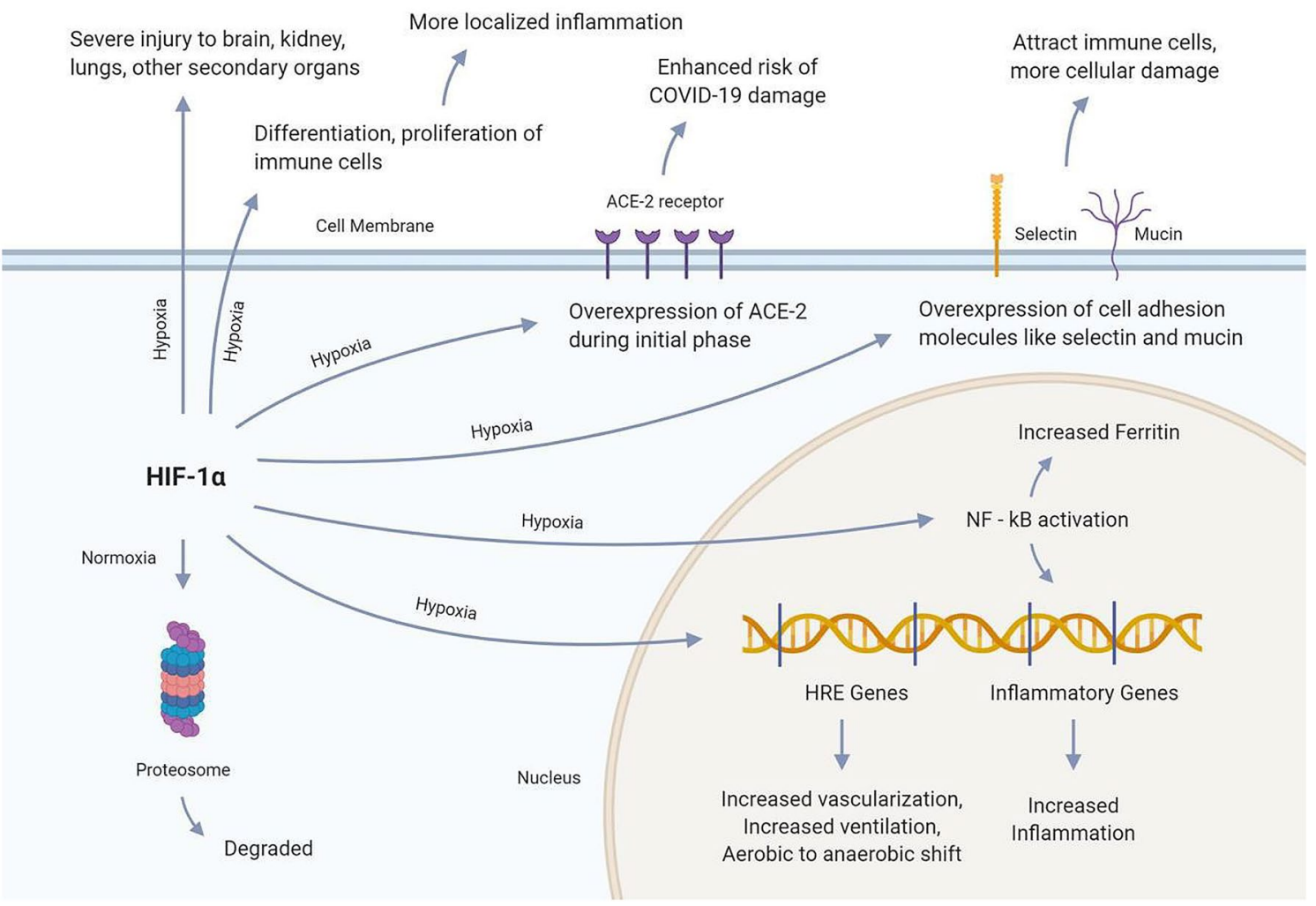

Fig. 1 Hypoxia Inducing Factor $1 \alpha$ is the prime mediator of hypoxia in cells. The factor under normal hypoxic conditions is degraded by the proteasome, therefore, the cell does not experience hypoxic symptoms. However, the factor can have several impacts on the hypoxic condition. HIF-1 $\alpha$ moves inside the nucleus and drives the transcription of HRE genes, which results in several hypoxic symptoms.
Besides, it may also drive the transcription of several inflammatory genes through NF-kB activation. NF-kB transcription factor activation has also been associated with increased serum ferritin accumulation. Moreover, HIF-1 $\alpha$ may increase the expression of the ACE-2 receptor on cells, suggesting an enhanced risk of COVID-19 infestation 


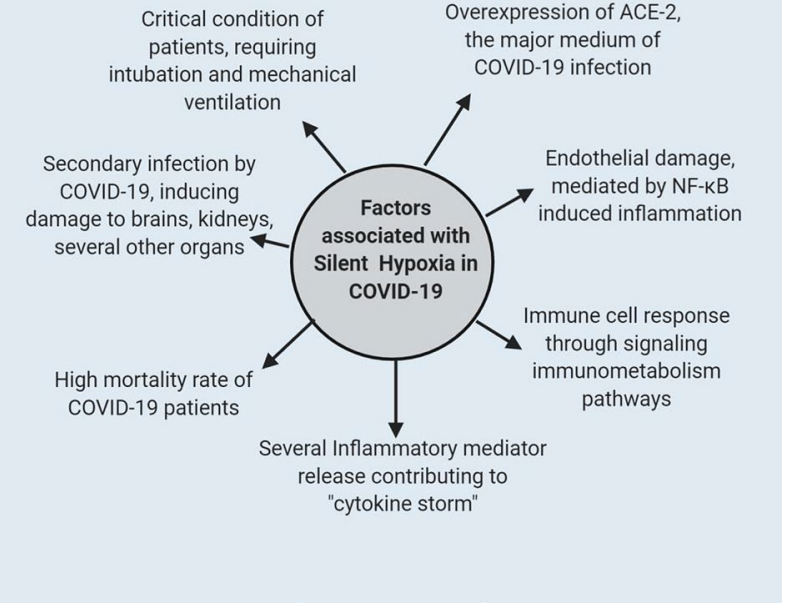

Fig. 2 Possible silent hypoxia associated factors involved in COVID19. Silent Hypoxia has been associated with several COVID-19 related symptoms. The condition can result in overexpression of ACE-2 receptors thereby increasing the risk of damage through COVID-19 infection. Besides, the condition can further contribute to the mechanism of "cytokine storm" by recruiting different mediators of inflammation. Moreover, silent hypoxia can cause serious endothelial damage through NF-kB transcription factor activation. Silent hypoxia can also signal a different immune-metabolism pathway and cause secondary organ damage. All these factors lead to the critical condition of patients along with an increased mortality rate among COVID-19 patients

Oxygen limitation impairs the mitochondrial oxidative phosphorylation pathway and inhibits the respiratory chain, which is the driving force of ATP generation within cells in the presence of oxygen. This impairment of the oxidative phosphorylation pathway results in an energy-deprived condition, an increase of proton leaks, and inhibition of important enzymes [20]. Compromised oxygen supply can severely affect the carotid bodies, neuro-epithelial bodies, and vascular smooth muscles. Besides, the respiratory centers consist of several chemoreceptor cells that function by sensing the $\mathrm{pH}$ level of the environment by detecting the concentration of hydrogen ions. The chemoreceptors involved in respiratory feedback are mainly categorized into central chemoreceptors and peripheral chemoreceptors. The central chemoreceptors are located on the ventrolateral surface of the medulla oblongata and are desensitized over time from mechanisms such as chronic hypoxia and enhanced carbon dioxide levels. Whereas the peripheral chemoreceptors can be either located on the aortic bodies or the carotid bodies. If peripheral chemoreceptors are located in the aortic bodies it does not detect $\mathrm{pH}$ changes rather detects imbalances in levels of blood oxygen and carbon dioxide. On the other hand, if the peripheral chemoreceptors are located on carotid bodies it detects imbalances in oxygen and carbon dioxide levels as well as $\mathrm{pH}$. Although the central chemoreceptors are desensitized over time due to stressful conditions, the peripheral chemoreceptors are not affected by these stress conditions but they have a lesser impact on the respiratory rate compared to central chemoreceptors [21]. These chemoreceptor cells lie in the synaptic contact with the branches of both afferent and efferent neurons and immediately transmit oxygen deprivation information to the respiratory centre of the brain by releasing neurotransmitters. Even after exerting so many instabilities within the cell, our cells become adapted to hypoxia by increasing the efficiency of energyproducing pathways and decreasing energy-consuming pathways [22].

Pneumonia is a major mediator of silent hypoxia in COVID-19 patients. Instead of filling air sacs with pus and fluid, the SARS-CoV-2 induced pneumonia causes air sacs to collapse (also referred to as "atelectasis"), which results in low oxygen supply in the body [23]. Studies aimed at unleashing the associated mechanism of air sac collapse in COVID-19 patients interpreted a sequence of events initiated by alveolar epithelial cell damage to surfactant dysfunction and alveolar instability, all of which leads to micro-atelectasis. These collapsed alveoli and air sacs are sealed by proliferating alveolar epithelial cells, which results in the formation of thickened septa and subsequent loss of alveolar function [24]. As the pulmonary system adapts for efficient oxygen transfer between pulmonary blood flow and alveolar ventilation even in differential oxygen pressure in different lung regions, COVID-19 associated silent hypoxia patients do not notice any vital signs of collapsed alveoli and air sacs. A combination of biological mechanisms takes place concurrently within the lung, which facilitates for this adjustment [25].

Nonetheless, this impact of HIF- $1 \alpha$ is temporary, and the expression of ACE-2 receptors has been found to decrease during the later stages of hypoxia. Moreover, this factor has been found to increase the expression of cell adhesion molecules like selectin and mucin, which attracts immune cells and worsen the degree of cellular damage. HIF- $1 \alpha$ is also associated with secondary organ damage, like the brain and kidney. Furthermore, this factor may trigger the differentiation and proliferation of the immune cells, which can result in more localized inflammation and tissue damage.

\section{Possible silent hypoxia associated factors involved in COVID-19 infection}

\section{Influence of HIF-1}

Hypoxia-Inducible Factor or HIF-1 is a dimeric transcription factor that acts as a master regulator of oxygen homeostasis in cells. This factor comprises of the HIF- $1 \alpha$ and 
HIF- $1 \beta$ subunits and is activated when the cell faces oxygen-depleted conditions [26]. Under normal cellular conditions, the HIF-1 remains deactivated. However, during the hypoxic conditions, this factor can subsequently interact with its co-activators and increase transcription of several hypoxia response elements (HRE) containing genes involved in increased ventilation, transferring the aerobic mechanisms to anaerobic, and improved vascularization $[21,26]$.

The HIF- $1 \alpha$ subunit has a potent effect on the expression of the ACE-2 gene [24]. ACE-2 is one of the major receptors that are targeted by the coronavirus to enter the human cell. Hypoxia has been found to increase the expression of the ACE-2 receptor gene during the early stages, which can worsen COVID-19 infection and increase damages to the lung cells. However, ACE-2 receptor expression remarkably reduces near baseline levels during the later stages of the condition. This is because the HIF-1 $\alpha$ upregulates the ACE promoter and causes an accumulation of angiotensin 2 enzyme, which in turn downregulates the ACE- 2 receptor expression [27]. Thus, this reduced expression of ACE-2 due to HIF-1 can be beneficial for reducing the risk of enhanced tissue and organ damage from COVID-19 infection.

\section{Hypoxia-Induced endothelial cell damage in COVID-19}

Hypoxia plays a central role in endothelial cell activation and adaptation by triggering different gene expression and cellular mechanisms. However, this influence of hypoxia in endothelium can be bidirectional. The HIF transcription factor can be either activated to support the integrity of the cell adhesion molecules or it can induce epithelial cell damage in an NF-kB mediated inflammation [28]. The destructive activation of endothelium cells correlates with pulmonary cell damage by recruiting different cytokines, chemokines, and pro-inflammatory mediators. Overexpression of HIF-1 has also been found to increase localized inflammation [26]. On the other hand, sometimes the cell expresses enhanced cell adhesion molecules like selectins or mucins and can recruit circulating endothelial cells to counter lung vascular injury, and, thereby exert a positive impact against lung damage [29]. The exact mechanism through which any one of these pathways can be induced by hypoxia is not well defined and needs further analysis.

\section{Role of hypoxia on innate and adaptive immunity in COVID-19 patients}

Hypoxia has the potential to drive different immune cell response through signaling a variety of immunometabolism pathways. The immune cells can be recruited for either a physiologically hypoxic environment that administers innate and adaptive immune response, or to the pathologically hypoxic environment that triggers immune cell dysfunction and tissue damage [30]. In the cases of COVID-19, some of the innate immune cells such as eosinophils, basophils, mast cells are considered to be the major mediators of the cytokine storm. Hypoxia can play a central role in driving the immune reactions from these cells and contribute to airway remodeling and inflammation [31]. The HIF-1 signaling pathway also upregulates the differentiation, proliferation, as well as survival of the innate and adaptive immune cells, thereby, producing an overwhelmed response against host cells. The mechanism of cytokine storm in COVID-19 is already known to cause irreparable damage to the lung cells and can be worse in response to these additional immune reactions generated by hypoxia-induced factors.

\section{Influence of viral infection in silent hypoxia}

A variety of viral pathogens have been found to influence the HIF-1 pathways in different ways to generate specified host responses such as promoting inflammation, altering metabolic pathways, down-regulating cellular defense mechanism, and facilitating viral replication. A recent study shows that Influenza H1N1 virus can activate the HIF-1 pathway and induce a hypoxic response in cells [32]. Although the influence of SARS-CoV-2 on inducing hypoxia has not been well investigated yet, some reports suggest a decreased count of hemoglobin and increased count of serum ferritin in COVID-19 patients [33]. This increased level of ferritin is a product of overexpression of HIF-1, which suggests SARS-CoV-2 can upregulate this transcription factor and induce hypoxia [34]. Besides, it has also been reported that the virus infection can induce lactic acid production and lactate dehydrogenase (LDH) enzyme production mediated by acute, uncompensated respiratory acidosis and sepsis. Respiratory acidosis is characterized by failure of metabolic compensation of acidosis and reduced plasma $\mathrm{pH}$ level without accompanying elevation of plasma bicarbonate levels. This decreased blood $\mathrm{pH}$ further leads to severe lung damage, hypercapnia and ultimately causes severe hypoxemia [35]. All this experimental evidence suggests a potential role of SARS-CoV-2 in triggering a hypoxic state of the lungs and greatly affecting lung physiology by triggering inflammation in COVID-19 infection.

\section{Other associated risk factors}

Silent hypoxia can induce indirect damage to the body organs, especially to the brain, by oxygen starvation and inflammation. Deceased COVID-19 patients very often have shown severe brain damage including stroke, hemorrhages, as well as acute encephalitis [36]. Moreover, silent hypoxia does not allow the patients to recognize the underlying symptoms of pulmonary destruction which are one major 
indicator of COVID-19 infection, making this condition even severe [36].

\section{Diagnosis of silent hypoxia associated COVID-19 patients}

Early detection of silent hypoxia in COVID-19 patients is crucial to minimize the long-term effect as well as mortality rate. Besides, early detection may facilitate deciding on when to start the oxygen treatment or whether to involve an invasive or non-invasive method of oxygenation. There are multiple ways one can find out the presence of silent hypoxia. Some of those procedures are jotted down below:

\section{Pulse oximeter}

A pulse oximeter is a common and standard medical device used to noninvasively measure the arterial oxygen saturation level and respiratory function in patients [37]. Although it represents an accessible device, it is important to ensure that the used pulse oximeter has a high accuracy rate, especially in cases when patient $\mathrm{SpO}_{2}$ is lower than $90 \%$ [38]. Constant monitoring of arterial oxygenation by pulse oximeter is a very good option to detect silent hypoxia. This device might be helpful for confirmed COVID-19 patients who are currently not demonstrating any severe symptoms of reduced oxygen saturation in the blood. Through constant monitoring, they might reduce the risk of complications that might appear suddenly due to silent hypoxia [39]. Moreover, alongside the saturation level of pulse oximeter, respiratory rate, heart rate, and NEWS 2 (National Early Warning Score) needed to be considered to unleash the silent hypoxic condition of patients [40].

\section{Blood gas analysis}

Arterial blood gas analysis is a process where a sample of blood is taken from the artery to check the concentration of different gases in the blood [41]. Right evaluation of blood sample through this method can provide a wide range of standard parameters such as partial pressure of oxygen and carbon dioxide, which are useful to investigate acidosis, alkalosis, as well as silent hypoxia [42, 43]. Recent studies report that alongside blood gas levels, $\mathrm{pH}$ level, bicarbonate, and electrolyte levels may also be utilized as an essential diagnostic factor for silent hypoxia in COVID-19 patients [44]. The increased partial pressure of carbon dioxide levels of confirmed COVID-19 patients may precede the measurement of $\mathrm{pH}$ levels and blood electrolyte levels to detect the presence of silent hypoxia. It has been reported that a comparatively lower level of $\mathrm{pH}$, calcium, sodium, and potassium are associated with disease severity and lower survival rates of COVID-19 patients [45, 46]. Blood gas analysis together with a pulse oximeter could be a valuable medium for the early detection of silent hypoxia in COVID19 patients [44].

\section{6-min walking test (6MWT)}

Silent hypoxia in COVID-19 patients can be detected by way of 6MWT, in which oxygen saturation level is measured after 6 min of taking walks. It has been reported that oxygen saturation level significantly reduced in COVID-19 patients in comparison to the control group [47]. According to a newly proposed modification in the $6 \mathrm{MWT}$ procedure aiming at the prognosis of viral load in mild COVID-19 patients compared to severe cases, the Single cutoff point for distance walked $<0.26$ mile ( $<1400$ feet)in 6 min (in terms of metabolic equivalent, equal to 3 METS) or $\mathrm{SpO}_{2}$ falling below $90 \%$ should be the definition of abnormality [48]. Moreover, patients with saturation levels falling by more $4 \%$ might also be considered severe cases. Those manifesting dyspnoea and fatigue without abnormality should be assessed based on other clinical comorbid conditions [49].

\section{Summary and recommendations}

The novel coronavirus pandemic caused by the SARSCoV-2 has affected people with different health conditions around the globe, but patients that develop silent hypoxia due to viral pathomechanism are possibly the worst sufferers. The underlying symptoms of a COVID-19 associated silent hypoxia slightly differ from that of normal hypoxia. Unlike normal hypoxic patients, patients with silent hypoxia do not experience any tachypnoea or dyspnoea. This does more damage to the patients by providing a short spanned feeling of comfort but long-lasting severe damage to the lungs. Several reports seem to suggest that silent hypoxia is well prevalent in COVID-19 patients but the reason behind this phenomenon is yet to be properly understood.

There are several factors associated with silent hypoxia and COVID-19 infection. The factor responsible for inducing hypoxic response, the HIF transcription factor, can cause the overexpression of ACE-2 receptors, serving as a medium of the entrance to the virus. Besides, the hypoxic condition can cause severe endothelial damage and contribute to the "cytokine storm", the characteristic feature of COVID-19 infection, further increasing the damage to body cells. Moreover, the virus also has the potential to increase localized inflammation through combined innate and adaptive immunity and cause the overexpression of HIF further inducing hypoxic condition in the lungs. All these responses can cause severe damage to several body tissues and ultimately lead to life-threatening situations in the COVID-19 patients. 
Since the patients with silent hypoxia do not feel any discomfort even when their oxygen saturation level is low, they need to be taken under a strict management process. Without continuous monitoring, it could turn out to be detrimental for the patient and might end up damaging respiratory organs and several other tissues. When a patient is diagnosed with COVID-19, he or she must go through a diagnosis of silent hypoxia immediately with a pulse oximeter, blood gas analysis, or other procedures. For proper management, COVID-19 patients diagnosed with silent hypoxia should be under intensive care including monitoring vital signs of disease severity, oxygen saturation levels, visual signs of discomfort, and deterioration in health conditions.

\section{Conclusion}

Based on the current knowledge, it is still unclear why silent hypoxia occurs in a COVID-19 patient. The available shreds of evidence suggest that the virus may be affecting the brain and nervous system, or there might be a lack of hypoxic vasoconstriction in such patients. However, other sources seem to suggest that hyper-fusion and normal carbon dioxide level even at the time of low oxygen saturation could be potential reasons behind this phenomenon. The clinicians are working to draw a solid conclusion on this matter. With efficient initiatives and further in-depth analysis, the underlying mechanism of this condition can be understood and better plans can be executed in the management of COVID19 patients with silent hypoxia.

Acknowledgement Authors are thankful to the members of the Community of Biotechnology and Swift Integrity Computational Lab, Dhaka, Bangladesh for the supports during the preparation of the manuscript.

Authors contributions YA conceived the study. YA, AR, TT, MU and MH designed the study. TT and AR wrote the draft manuscript. MH, YA, AN and MU edited and revised the manuscript. All the authors approved the final version of the manuscript.

Funding No specific grant was received for this study.

Data availability All the data are provided within the manuscript.

\section{Declarations}

Conflict of interest The authors declare that the research was conducted in the absence of any commercial or financial relationships that could be construed as a potential conflict of interest.

\section{References}

1. Coronavirus disease (COVID-19) - World Health Organization [Internet]. Who.int. 2020 [cited 2 September 2020]. Available from: https://www.who.int/emergencies/diseases/novel-coron avirus 2019

2. Coronavirus Update (Live): $133,450,721$ Cases and 2,892,333 Deaths from COVID-19 Virus Pandemic-Worldometer [Internet]. Worldometers.info. 2021 [cited 7 April 2021]. Available from: https://www.worldometers.info/coronavirus/

3. Rothan H, Byrareddy S (2020) The epidemiology and pathogenesis of coronavirus disease(COVID-19) outbreak. J Autoimmun 109:102433

4. Lu R, Zhao X, Li J, Niu P, Yang B, Wu H et al (2020) Genomic characterization and epidemiology of 2019 novel coronavirus: implications for virus origins and receptor binding. The Lancet 395(10224):565-574

5. Zou X, Chen K, Zou J, Han P, Hao J, Han Z (2020) Single-cell RNA-seq data analysis on the receptor ACE2 expression reveals the potential risk of different human organs vulnerable to 2019nCoV infection. Frontiers of Medicine 14(2):185-192

6. Ullah M, Islam H, Rahman A, Masud J, Shweta D, Araf Y et al (2020) A generalized overview of SARS-CoV-2: where does the current knowledge stand. Electron J General Med 17(6):em251

7. Contributor S. 'Silent hypoxia' may be killing COVID-19 patients. But there's hope. [Internet]. livescience.com. 2020 [cited 2 September 2020]. Available from: https://www.livescience.com/ silent-hypoxia-killing-covid-19-coronavirus-patients.html

8. Levitan R (2020) Pulse oximetry as a biomarker for early identification and hospitalization of COVID-19 pneumonia. AcadEmerg Med 27(8):785-786

9. Decoding silent hypoxia in Covid patients: Blue lips, changing skin colour, sweating for no reason [Internet]. The Economic Times. 2020 [cited 28 March 2021]. Available from: https://econo mictimes.indiatimes.com/magazines/panache/decoding-silenthypoxia-in-covid-patients-blue-lips-changing-skin-colour-sweat ing-for-no-reason/articleshow/80009671.cms

10. Emily Henderson B. Silent hypoxia and its role in COVID-19 detection [Internet]. News-Medical.net. 2020 [cited 2 September 2020]. Available from: https://www.newsmedical.net/news/20200 603/Silent-hypoxia-and-its-role-in-COVID-19-detection.aspx

11. Harun M, Hossain M, Bari M, Rubel N, Karim M, Siddiquee $\mathrm{N}$ et al (2020) Pulse oximetry is essential in home management of elderly COVID-19 patients. Bangladesh J Otorhinolaryngol 26(1):55-67

12. Happy hypoxia in COVID-19: New study may have found possible causes behind this biology-defying complication-Health News,Firstpost [Internet]. Firstpost. 2020 [cited 2September 2020]. Available from: https://www.firstpost.com/health/happyhypoxia-incovid-19-new-study-may-have-found-possible-causesbehind-this-biology-defyingcomplication-8578751.html

13. What is 'silent hypoxia'? The coronavirus symptom patients don't know they have [Internet]. Global News. 2020 [cited 2 September 2020]. Available from: https://globalnews.ca/news/6930367/ silent-hypoxia-coronavirus/

14. 5.4: Signs and Symptoms of Hypoxia [Internet]. Medicine LibreTexts. 2020 [cited 2 September 2020]. Available from: https://med.libretexts.org/Bookshelves/Nursing/Book\%3A_Clini cal_Procedures_for_Safer_Patient_Care_(Doyle_and_McCut cheon)/05\%3A_Oxygen_Therapy/5.04\%3A_Signs_and_Sympt oms_of_Hypoxia\#: :text=Early $\% 20$ signs $\% 20$ of $\% 20$ hypoxia\% 20are,level\%20of\%20consciousness\%20will\%20decrease.

15. (COVID-19) C, Health E, Disease H, Disease L, Management P, Conditions $\mathrm{S}$ et al. Hypoxia and Hypoxemia: Symptoms, Treatment, Causes [Internet]. WebMD. 2020 [cited 2 September 2020]. Available from: https://www.webmd.com/asthma/guide/hypox iahypoxemia\#: : :text=Hypoxemia\% 20 (low\% 20oxygen $\% 20$ in $\%$ 20your,used $\% 20$ to $\% 20$ describe $\% 20$ both $\% 20$ problems.

16. H. Happy hypoxia in patients worries doctors [Internet]. The New Indian Express. 2020 [cited 2 September 2020]. Available from: 
https://www.newindianexpress.com/states/karnataka/2020/aug/05/ happy-hypoxia-inpatients-worries-doctors-2179175.html

17. Tobin M, Laghi F, Jubran A (2020) Why COVID-19 silent hypoxemia Is baffling to physicians. Am J RespirCrit Care Med 202(3):356-360

18. Post T. Patients who died of COVID-19 in Banyumas experienced 'happy hypoxia' [Internet]. The Jakarta Post. 2020 [cited 2 September 2020]. Available from: https://www.thejakartapost.com/ news/2020/08/20/patients-who-died-of-covid-19-inbanyumasexperienced-happy-hypoxia.html

19. Fuglebjerg N, Jensen T, Hoyer N, Ryrs $\varnothing$ C, Madsen B, Harboe Z (2020) Silent hypoxia in patients with SARS CoV-2 infection before hospital discharge. Int J Infect Dis 99:100-101

20. Gnaiger E (2001) Bioenergetics at low oxygen: dependence of respiration and phosphorylation on oxygen and adenosine diphosphate supply. RespirPhysiol 128(3):277-297

21. Marieb E, Hoehn K (2013) Chapter 22: The respiratory system. In: Human anatomy and physiology, 9th ed. Pearson, pp 801-841

22. Michiels C (2004) Physiological and pathological responses to hypoxia. Am J Pathol 164(6):1875-1882

23. Teo J (2020) Early detection of silent hypoxia in Covid-19 pneumonia using smartphone pulse oximetry. J Med Syst 44(8):134

24. Ochs M, Timm S, Elezkurtaj S, Horst D, Meinhardt J, Heppner F et al (2021) Collapse induration of alveoli is an ultrastructural finding in a COVID-19 patient. EurRespir J 14:2004165

25. Weissmann N (2008) Hypoxia-driven mechanisms in lung biology and disease: a new review series of the ERS Lung Science Conference. EurRespir J 31(4):697-698

26. Jennifer E. Ziello Y. Hypoxia-Inducible Factor (HIF)-1 Regulatory Pathway and its Potential for Therapeutic Intervention in Malignancy and Ischemia [Internet]. PubMed Central (PMC). 2020 [cited 28 August 2020]. Available from: https://www.ncbi.nlm. nih.gov/pmc/articles/PMC2140184/

27. Zhang R, Wu Y, Zhao M, Liu C, Zhou L, Shen S et al (2009) Role of HIF- $1 \alpha$ in the regulationACE and ACE2 expression in hypoxic human pulmonary artery smooth muscle cells. Am J Physiol Lung Cell MolPhysiol 297(4):L631-L640

28. Marchetti M (2020) COVID-19-driven endothelial damage: complement, HIF-1, and ABL2 are potential pathways of damage and targets for cure. Ann Hematol 99(8):1701-1707

29. Gao X, Chen W, Liang Z, Chen L (2011) Autotransplantation of circulating endothelial progenitor cells protects against lipopolysaccharide-induced acute lung injury in rabbit. IntImmunopharmacol 11(10):1584-1590

30. Taylor C, Colgan S (2017) Regulation of immunity and inflammation by hypoxia in immunological niches. Nat Rev Immunol 17(12):774-785

31. Dahlin J, Hallgren J (2020) Mast cell progenitors: origin, development and migration to tissues. MolImmunol 63(1):9-17

32. Ren L, Zhang W, Han P, Zhang J, Zhu Y, Meng X et al (2019) Influenza A virus (H1N1) triggers a hypoxic response by stabilizing hypoxia-inducible factor- $1 \alpha$ via inhibition of proteasome. Virology 530:51-58

33. Gómez-Pastora J, Weigand M, Kim J, Wu X, Strayer J, Palmer A et al (2020) Hyperferritinemia in critically ill COVID-19 patients-is ferritin the product of inflammation or a pathogenic mediator? ClinChimActa 509:249-251

34. Chen T, Wu D, Chen H, Yan W, Yang D, Chen G et al (2020) Clinical characteristics of 113 deceased patients with coronavirus disease 2019: retrospective study. BMJ 368:m1091
35. Traylor Z, Aeffner F, Davis I (2020) Influenza A H1N1 induces declines in alveolar gas exchange in mice consistent with rapid post-infection progression from acute lung injury to ARDS. Influenza Other Respir Viruses 7(3):472-479

36. Cormier Z. How Covid-19 can damage the brain [Internet]. Bbc. com. 2020 [cited 28 August 2020]. Available from: https://www. bbc.com/future/article/20200622-the-longterm-effects-of-covid19-infection

37. Jubran A (2004) Pulse oximetry. ApplPhysiol Intensive Care Med 30(11):29-32

38. Quaresima V, Ferrari M (2020) COVID-19: efficacy of prehospital pulse oximetry for early detection of silent hypoxemia. Crit Care 24(1):501

39. Luks A, Swenson E (2020) Pulse oximetry for monitoring patients with covid at home. Potential pitfalls and practical guidance. Annals Am ThoracSoc 17(9):1040-1046

40. National Health Service (NHS) England. Pulse oximetry to detect early deterioration of patients with COVID-19 in primary and community care settings [Internet]. [Accessed 2 July 2020]. Available from: https://www.england.nhs.uk/coronavirus/publication/ pulse-oximetry-to-detect-early-deterioration-ofpatients-withcovid-19-in-primary-and-community-care-settings/

41. Pulmonary Function Testing [Internet]. Temple Health. 2020 [cited 2 September 2020]. Available from: https://www.templ ehealth.org/services/treatments/pulmonary-functiontesting

42. Arterial HD (2019) Arterial blood gas interpretation demystified. Phys Assist Clin 4(3):551-560

43. Narayan R, Wang M, Laurencin C, Yu X, Hellmich C, Krishnan S et al (2018) Encyclopedia of biomedical engineering. University of Nort Carolina, Chapel hill

44. Elezagic D, Johannis W, Burst V, Klein F, Streichert T. Venous blood gas analysis in patients with COVID-19 symptoms in the early assessment of virus positivity. LaboratoriumsMedizin. 2020;0(0).

45. Skevaki C, Fragkou P, Cheng C, Xie M, Renz H (2020) Laboratory characteristics of patients infected with the novel SARSCoV-2 virus. J Infect 81(2):205-212

46. Lippi G, South A, Henry B (2020) Electrolyte imbalances in patients with severe coronavirus disease 2019 (COVID-19). Annals ClinBiochem Intern J Lab Med 57(3):262-265

47. RE: COVID-19, on the edge of hypoxia [Internet]. Science. 2020 [cited 2 September 2020]. Available from: https://science.scien cemag.org/content/re-covid-19-edge-hypoxia

48. Shephard R (2012) Validity of the 6min walk test in prediction of the anaerobic threshold before major non-cardiac surgery. Yearbook of Sports Medicine 2012:172-174

49. Mantha S, Tripuraneni S, Roizen M, Fleisher L (2020) Proposed modifications in the 6-minute walk test for potential application in patients with mild COVID-19: a step to optimize triage guidelines. AnesthAnalg 131(2):398-402

Publisher's Note Springer Nature remains neutral with regard to jurisdictional claims in published maps and institutional affiliations. 